\title{
Kinetic Monte Carlo simulations of oscillatory shape evolution for electromigration-driven islands
}

\author{
Marko Rusanen, ${ }^{1}$ Philipp Kuhn, ${ }^{2}$ and Joachim $\mathrm{Krug}^{2}, *$ \\ ${ }^{1}$ Laboratory of Physics, Helsinki University of Technology, \\ P.O. Box 1100, FI-02015 TKK, Espoo, Finland \\ ${ }^{2}$ Institut für Theoretische Physik, Universität zu Köln, Zülpicher Strasse 77, D-50937 Köln, Germany
}

(Dated: August 6, 2018)

\begin{abstract}
The shape evolution of two-dimensional islands under electromigration-driven periphery diffusion is studied by kinetic Monte Carlo (KMC) simulations and continuum theory. The energetics of the KMC model is adapted to the $\mathrm{Cu}(100)$ surface, and the continuum model is matched to the KMC model by a suitably parametrized choice of the orientation-dependent step stiffness and step atom mobility. At $700 \mathrm{~K}$ shape oscillations predicted by continuum theory are quantitatively verified by the KMC simulations, while at $500 \mathrm{~K}$ qualitative differences between the two modeling approaches are found.

PACS numbers: 68.65.-k, 66.30.Qa, 05.45.-a, 05.10.Ln
\end{abstract}

\section{INTRODUCTION}

The nonequilibrium evolution of two-dimensional nanoscale structures is a central theme in current surface science, which has been the focus of many experimental and theoretical studies $1,2,3,4,5$. On the theoretical side, a key challenge is to bridge the length and time scales between individual atomic processes and the mesoscopic step morphologies that they produce ${ }^{4.6}$. A highly successful approach to this problem treats the crystal steps as continuous entities, whose dynamics is governed by the interplay between the adatom population on the terraces, and the processes at the steps which define effective boundary conditions on the adatom density field ${ }^{1.4 .7}$. This approach is well established when the steps are rough and sufficiently close to equilibrium for thermodynamic considerations to apply at least locally. However, atomistic details not captured in the continuum theory must generally be expected to become important at low temperatures and under strong driving 8.9.10.11.

In this paper we compare atomistic and continuous modeling strategies for the specific case of island electromigration ${ }^{12,13,14}$. The present interest in electromigration-induced surface morphology evolution is motivated both by its importance for the reliability of integrated $\operatorname{circuits}^{15}$, and by its potential for a rich variety of nonlinear dynamic phenomena. Specifically, it has recently been shown within a continuum approach that the motion and shape evolution of electromigrationdriven islands in the regime dominated by periphery diffusion (PD) can be surprisingly complex when the crystal anisotropy of the mobility of atoms along the island edge is taken into account ${ }^{16,17}$. Depending on the island size and the strength of the anisotropy, a range of different dynamic phases involving spontaneous symmetry breaking as well as oscillatory and chaotic shape evolution has been predicted.

With regard to a possible experimental observation of these phases, it is important to understand to what ex- tent the features of the continuum model survive the discreteness and stochasticity introduced into the dynamics on the atomic level. We have therefore conducted extensive Kinetic Monte Carlo (KMC) simulations of island electromigration, using a realistic model of the $\mathrm{Cu}(100)$ surface $^{18}$. At high temperatures $(T=700 \mathrm{~K})$ we find good agreement between the continuum and discrete models, and report for the first time (to the best of our knowledge) a case of oscillatory shape evolution within a fully stochastic KMC simulation. At lower temperatures the oscillations persist, but additional features not covered by the continuum description appear. The discrete and continuum models used in this work are described in the next Section. Results at high and low temperature are presented in Sect III and a concluding discussion can be found in Sect[V]

\section{MODELS}

\section{A. Kinetic Monte Carlo model}

Our KMC simulations are based on a lattice-gas model ${ }^{18}$ with energetics obtained from effective medium theory ${ }^{19}$ (EMT). The energy barriers of the model and their relative ordering are in good agreement with experimental data for the $\mathrm{Cu}(001)$ surface $^{2}$. It should be noted that, within the EMT description, barriers on the $\mathrm{Ag}(001)$ and $\mathrm{Ni}(001)$ surfaces are very similar to those of $\mathrm{Cu}(001)$ up to an overall scaling factor ${ }^{19}$. The simulations were implemented using the rejection-free Monte Carlo algorithm by Bortz et al. ${ }^{20}$ with a binary tree structure ${ }^{21}$. This allowed us to reach time scales on the order of seconds in physical time and island sizes up to 10000 atoms within reasonable computation time. Our model is very similar to that used by Mehl et al ${ }^{13}$, who also considered the $\mathrm{Cu}(100)$ surface. However, working at somewhat lower temperatures and with smaller islands, they did not observe the complex phenomena described 
in the present paper.

In principle, the KMC model includes all relevant oneparticle processes and their corresponding energy barriers as given in Ref.19. However, since our goal is to compare the KMC simulations with the continuum theory, we need to exclude some processes from the KMC simulations. Since in the continuum model the total mass of an island is conserved, we explicitly forbid all adatom detachment events from an island. This does however not exclude a jump into a site without any nearest neighbors (NN) but with at least one next nearest neighbor atom. This local restriction permits both atoms to jump around corner sites and excitation of an atom at the boundary with three NN's into an edge atom with one NN through two jumps. We also exlude vacancy diffusion inside the island, as this is not included in the continuum model.

It must be emphasized that these restrictions do not violate the detailed balance condition in our simulations. We have checked that the oscillatory shape evolution phenomena described in this paper persist even when they are relaxed. Needless to say, however, the definition of the island size becomes problematic for long times when detachment of atoms from the island is allowed for.

The KMC simulations are conducted as follows. Initially an island with a given number of atoms $S$ is placed on a lattice in a square or rectangular configuration. The hopping rate $\nu$ of an atom to a vacant NN site on the island edge is given by

$$
\nu=\nu_{0} \exp \left[-\left(E_{S}-\min \left(0, \Delta_{N N}\right) E_{B}\right) / k_{\mathrm{B}} T\right],
$$

where the attempt frequency $\nu_{0}=3.0 \times 10^{12} \mathrm{~s}^{-1}$ and the barrier for the jump of an atom at a straight edge is $E_{S}=$ $0.26 \mathrm{eV}$. The change in bond number $-3 \leq \Delta_{N N} \leq 3$ is the difference in the number of $\mathrm{NN}$ bonds between the initial and final states with bond energy $E_{B}=0.26 \mathrm{eV}$. Thus, the model gives an additional kink rounding barrier $E_{\mathrm{kr}}=0.26 \mathrm{eV}$ (compared to the diffusion along the close-packed [110] edges) and a total detachment barrier $E_{\mathrm{det}}=0.52 \mathrm{eV}$ from a kink site onto a edge site.

The electromigration force is introduced by decreasing (increasing) the barrier for hops along (against) the direction of the force by an amount $E_{\text {bias. }}$. In most of the simulations described here the force was directed along the $x$-direction of the lattice. Experimentally realizable values of the bias $\frac{13.16}{}$ are on the order of $E_{\text {bias }} \approx 10^{-5}$ $\mathrm{eV}$ for $\mathrm{Cu}(100)$ at a current density of $10^{7} \mathrm{~A} / \mathrm{cm}^{2}$. The values used in the simulations are typically two orders of magnitude larger. However, at least in certain regimes it is easy to extrapolate to realistic bias strengths by considering larger islands (see the discussion below in Sect

\section{B. Continuum model}

The continuum description of two-dimensional shape evolution by $\mathrm{PD}$ is based on the continuity equation 14.16 .17

$$
v_{n}+\frac{\partial}{\partial s} a^{2} \sigma\left[-\frac{\partial}{\partial s}\left(a^{2} \tilde{\gamma} \kappa\right)+F_{t}\right]=0
$$

for the normal velocity $v_{n}$ of the island boundary. Here $s$ denotes the arclength, $a$ the distance between NN sites, $\sigma$ the step atom mobility, $\tilde{\gamma}$ the edge stiffness, $\kappa$ the local curvature, and $F_{t}$ the tangential component of the electromigration force acting on an edge atom. We take the force to be of constant strength $F_{0}$ and aligned along the $x$-axis. With $\theta$ denoting the angle between the normal of the island edge and the $y$-axis (counted positive in the clockwise direction), this implies $F_{t}=F_{0} \cos (\theta)$.

The crystal anisotropy of the surface enters the continuum model through the dependence of $\tilde{\gamma}$ and $\sigma$ on the edge orientation $\theta$. In previous work based on (2) the stiffness was assumed to be isotropic, and a simple model expression with sixfold symmetry was used for the mobility16.17. Here we are more ambitious, and aim to adapt the functions $\tilde{\gamma}(\theta)$ and $\sigma(\theta)$ as closely as possible to the thermodynamics and kinetics of the KMC model.

The orientation dependence of the step stiffness on fcc(100) surfaces, in particular on $\mathrm{Cu}(100)$, has been of considerable recent interest 23.24 . For our purposes it is sufficient to use the simple expression

$$
\tilde{\gamma}(\theta)=\frac{k_{\mathrm{B}} T}{a} \frac{\left(1+m^{2}\right)^{3 / 2}}{m^{2}+\sqrt{m^{2}+\left(a / l_{k}\right)^{2}}}, \quad m=\tan (\theta),
$$

where $l_{k}=(a / 2) e^{\epsilon / k_{\mathrm{T}}}$ is the distance between thermally excited kinks, and $\epsilon=E_{B} / 2=0.13 \mathrm{eV}$ is the kink energy. Equation (3) interpolates in a natural way between the leading order terms obtained in a low temperature expansion of the full expression for the two-dimensional Ising lattice gas for $m>0$ and $m=0$, respectively. For the close-packed orientation $m=0$ it reduces to the wellknown relation ${ }^{1,2.3}$

$$
\tilde{\gamma}(0)=\frac{k_{\mathrm{B}} T}{a^{2}} l_{k}=\frac{k_{\mathrm{B}} T}{2 a} e^{\epsilon / k_{\mathrm{T}}}
$$

while for $m \neq 0$ and $T \rightarrow 0$ one obtains the purely entropic low-temperature expression ${ }^{23}$

$$
\tilde{\gamma}(\theta)=\frac{k_{\mathrm{B}} T}{a} \frac{\left(1+m^{2}\right)^{3 / 2}}{m^{2}+|m|} .
$$

The transition between the two regimes occurs, and the singularity of (5D) is cut off, when the concentration $m$ of forced kinks becomes comparable to the concentration $a / l_{k}$ of thermal kinks.

The anisotropy of the mobility is less well understood, and experimental data are so far scarce $\frac{25.26}{}$. It is known that $\sigma$ is isotropic in the absence of an additional kink rounding barrier 10.27 . Here we extend a phenomenological expression previously derived for the close-packed step orientation ${ }^{9.28}$ to general orientations by writing

$$
\sigma(\theta)=\frac{a \nu_{0} e^{-E_{\mathrm{det}} / k_{\mathrm{B}} T}}{\left(k_{\mathrm{B}} T\right)\left[1+\left(a / l_{\mathrm{eff}}(\theta)\right) p_{\mathrm{kr}}^{-1}\right]},
$$


where $p_{\mathrm{kr}}=e^{-E_{\mathrm{kr}} / k_{\mathrm{B}} T}$ is the kink rounding probability. The effective kink spacing $l_{\text {eff }}$, which replaces the thermal kink spacing $l_{k}$ at nonzero tilts, is defined in terms of the stiffness (given by (3)) through the relation

$$
l_{\text {eff }}(\theta)=\left(a^{2} / k_{\mathrm{B}} T\right) \tilde{\gamma}(\theta) .
$$

This is motivated by the general expression for the roughness of a step configuration $\zeta(x)$,

$$
\left\langle\left(\zeta(x)-\zeta\left(x^{\prime}\right)\right)^{2}\right\rangle=\frac{k_{\mathrm{B}} T}{\tilde{\gamma}}\left|x-x^{\prime}\right|,
$$

which is valid for steps of arbitrary orientation ${ }^{1.2 .3}$. On the basis of (8) we define the effective kink spacing as that length scale at which the amplitude of step fluctuations becomes equal to the NN lattice spacing. Equating the right hand side of (8) to $a^{2}$ then immediately leads to (7).

The dependence of the mobility (6) on temperature and orientation is fully specified by the values of the kink energy and the kink rounding barrier, and reproduces correctly the behavior in various known limits. For $\theta=0$ the low temperature behavior of the mobility depends ${ }^{28}$ on whether the kink rounding barrier is weak (in the sense of $E_{\mathrm{kr}}<\epsilon$ ) or strong $\left(E_{\mathrm{kr}}>\epsilon\right.$ ). In the first case $\sigma(0) \sim e^{-E_{\mathrm{det}} / k_{\mathrm{B}} T}$, while in the second case $\sigma(0) \sim e^{-\left(E_{\mathrm{det}}+E_{\mathrm{kr}}-\epsilon\right) / k_{\mathrm{B}} T}$. For kinked steps $l_{\mathrm{eff}} / a$ is of order unity, and hence $\sigma \sim e^{-\left(E_{\mathrm{det}}+E_{\mathrm{kr}}\right) / k_{\mathrm{B}} T}$, in accordance with Monte Carlo simulations $\frac{10}{}$. For the case of a strong kink rounding barrier, which is realized in our system, the anisotropy of the mobility [the ratio between the maximum and minimum values of $\sigma(\theta)]$ is thus of the order of $e^{\epsilon / k_{\mathrm{B}} T}$, which is comparable to the anisotropy of the stiffness. A detailed comparison of (6) with numerical measurements of the mobility within the KMC model will be presented elsewhere.

The continuum model (2) can be made dimensionless by scaling lengths with

$$
l_{E}=\sqrt{a^{2} \tilde{\gamma}(0) / F_{0}}
$$

and time with

$$
t_{E}=\frac{l_{E}^{4}}{\left[\sigma(0) \tilde{\gamma}(0) a^{4}\right]}
$$

This implies in particular that increasing the electromigration force is equivalent (up to a rescaling of time) to an increase of the island area by the same factor. Generally speaking, electromigration effects become important when the linear island size is comparable to $l_{E}$. When presenting the results for the continuum model the island size will be parametrized by the dimensionless radius $\hat{R}$ of a circle of the same area, and time is measured in units of $t_{E}$. For comparison with the KMC simulations, the dimensionless radius of an islands of $S$ atoms is then defined by $\hat{R}=\sqrt{S / \pi} / l_{E}$.

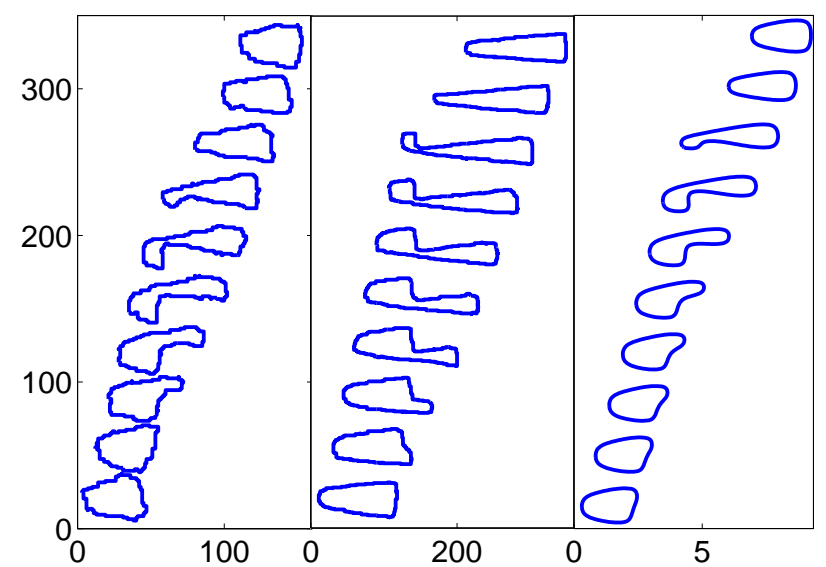

FIG. 1: Time sequences of island configurations obtained from KMC simulations (left and middle columns) and numerical solution of the continuum equation (rightmost column). Note that all configurations are displaced in the $y$-direction linearly in time. Parameters are $T=700 \mathrm{~K}, S=1000, E_{\text {bias }}=$ $2 \times 10^{-3} \mathrm{eV}$ (left column), $T=500 \mathrm{~K}, S=4000, E_{\text {bias }}=10^{-3}$ $\mathrm{eV}$ (middle column), $T=700 \mathrm{~K}, \hat{R}=2.35$ (right column).

\section{RESULTS}

\section{A. $700 \mathrm{~K}$}

In Figure 1 we show snapshots of the island shape obtained from the KMC simulations (left panel at $T=700$ $\mathrm{K}$, middle at $T=500 \mathrm{~K}$ ) in comparison with the continuum model results at $700 \mathrm{~K}$ (right panel). Apart from the shape fluctuations associated with the discreteness of the KMC model, the shape evolution at $700 \mathrm{~K}$ is seen to be very similar to that predicted by the continuum model. The dimensionless radius corresponding to the KMC simulation parameters is $\hat{R}=1.56$, somewhat smaller than the value $\hat{R}=2.35$ used in the continuum calculations.

In the KMC simulations an oscillation starts as random protrusions of the front edge of the island boundary grow large enough to be amplified by the electromigration force. The front edge effectively splits into two parts, and a straight segment perpendicular to the field appears on the island boundary, which is left behind as the protrusion advances. In the reference frame of the island the standing segment moves backwards and eventually reaches the back end of the island. At this point the island has elongated to about twice its initial length. When the standing segment disappears the island therefore contracts back into a quasi-stationary shape, from which the next oscillation is triggered. The whole sequence of events is identical to that observed in the continuum model. The formation of a standing boundary segment corresponds precisely to the static facets found previously for islands of sixfold symmetry ${ }^{16}$.

It can be seen from Fig 1 that the shape oscillations break the symmetry with respect to the horizontal (the 


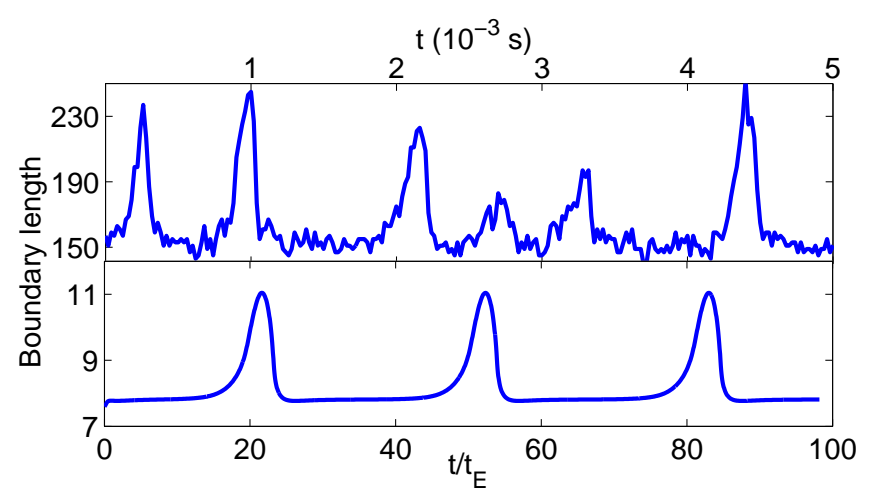

FIG. 2: Oscillations of the boundary length obtained from KMC simulations (upper panel) and the continuum model (lower panel). The conditions correspond to the left and right columns of Fig 1 ]

direction of the electromigration force), since the initial protrusion may appear either in the upper or the lower part of the front edge. The continuum model is strictly deterministic. Hence the direction of symmetry breaking is fixed by the initial condition, and each oscillation event causes a vertical shift of the center of mass of the island in the same direction. This implies that the mean direction of island motion forms a nonzero angle with the direction of the force, and places this mode of migration into the oblique oscillatory (OO) phase that was previously identified in the case of sixfold symmetry ${ }^{16}$. In contrast, in the KMC simulations the direction changes randomly from one oscillation event to the next. At least for the time sequences of limited length that were generated in our simulations, no correlations between subsequent events could be detected, and hence the island center of mass performs a random walk in the transverse direction.

Continuum calculations carried out over a range of dimensionless island radii show that the $\mathrm{OO}$ phase is sandwiched between a narrow regime of oblique stationary (OS) motion (where islands of stationary shape move at a small angle with respect to the direction of the force) for $2.16<\hat{R}<2.24$, and a regime where islands break up for $\hat{R} \geq 2.4$. For $\hat{R} \leq 2.16$ the motion is stationary and straight in the direction of the force (the SS phase). Apart from a shift in the locations of the phase boundaries, this is in agreement with the KMC simulations, where regimes of stationary and oscillatory behavior followed by island breakup were observed with increasing island size. The OS regime could not be unambiguously identified in the KMC simulations, possibly because the small obliqueness in the direction of motion is easily lost in the fluctuations.

Quantitatively the oscillation of the island shape can be monitored by various observables such as the boundary length of the island, the average island velocity or the center of mass coordinates. In Figure 2 we show the evo-

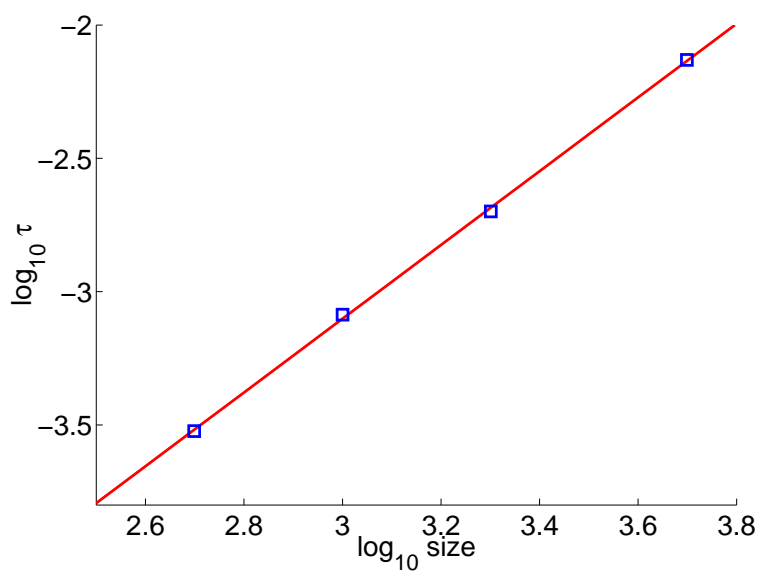

FIG. 3: Oscillation period (in units of seconds) measured in KMC simulations for islands of size $S=500-5000$ and bias strengths $E_{\text {bias }}=4 \times 10^{-3} \mathrm{eV}-4 \times 10^{-4} \mathrm{eV}$, such that the dimensionless island radius is $\hat{R}=1.56$ in all cases.

lution of the boundary length corresponding to the time sequences depicted in Figure 1. Despite a large amount of irregularity in the timing of the oscillations observed in the KMC simulations, a typical period is clearly visible, and the overall shape and amplitude of the individual events is quite similar to those obtained from the continuum model. For the parameters of the KMC simulation the characteristic time scale is $t_{E} \approx 1.3 \times 10^{-4} \mathrm{~s}$, which is a factor 2.6 larger than the scale used to match the two time sequences in Fig 2 Since $t_{E} \sim l_{E}^{4}$, this discrepancy is comparable to that found above in the comparison of the effective island radius $\hat{R}$. In view in particular of the uncertainties associated with the phenomenological expression (6) for the step atom mobility, the agreement between the two models must be considered remarkably good.

Within the continuum model the behavior of the island depends only on the dimensionless island size $\hat{R}$, defined relative to the characteristic length $l_{E}$ in (9). To check if this property carries over to the discrete model, we performed a series of KMC simulations in which the island size and the strength of the bias were varied in such a way that the product $S E_{\text {bias }}$ remained constant, and which therefore correspond to a single value of the dimensionless island radius $\hat{R}$. As predicted by continuum theory, the same kind of oscillations depicted in the first column of Fig 1 was observed for island sizes between $S=500$ and $S=5000$. In addition, the oscillation period $\tau$ was found to scale with island size as a power law,

$$
\tau \sim S^{z}
$$

with $z \approx 1.4$ (Fig 3). The measured exponent $z$ is somewhat smaller than the value $z=2$ expected from continuum theory. There is however a substantial uncertainty in the estimates of $\tau$ both for large islands, where only 


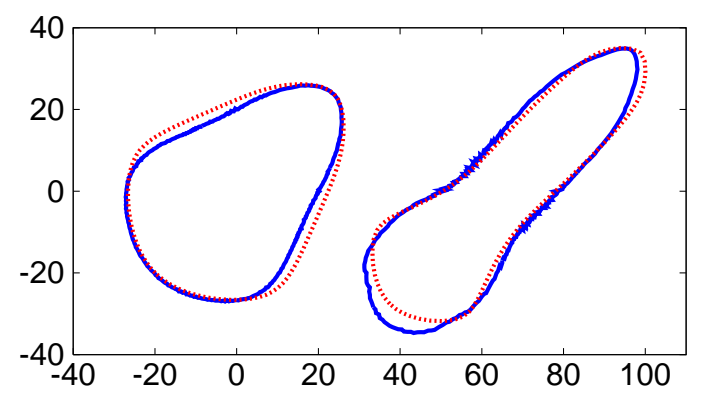

FIG. 4: Stationary island shapes obtained at $T=700 \mathrm{~K}$ with the electromigration force directed along the lattice diagonal. Full blue lines show time-averaged KMC shapes for island size $S=2000$ and bias $E_{\text {bias }}=5 \times 10^{-4} \mathrm{eV}$ (left) and $E_{\text {bias }}=$ $10^{-3} \mathrm{eV}$ (right). Dotted red lines show stationary continuum shapes of dimensionless radius $\hat{R}=1.9$ (left) and $\hat{R}=2.9$ (right).

a small number of oscillations could be observed, and for small islands, where the oscillations are obscured by noise.

Exploratory simulations in which the direction of the electromigration force was chosen to be different from the lattice axes indicate that the oscillatory behavior generally persists. An exception is the case when the force points along the lattice diagonal, where no oscillations are observed. Instead, the islands attain a characteristic shape which becomes increasingly elongated with increasing island size or bias strength. This behavior is well reproduced by the continuum model. In Fig 4 the timeaveraged shapes obtained from the KMC simulations are compared to stationary shapes generated by the continuum model. The match between the two sets of shapes is seen to be excellent, provided some adjustement of the effective islands size is allowed for. The parameters of the KMC simulations in Fig 4 imply dimensionless radii of $\hat{R}=1.106$ (left) and $\hat{R}=1.56$ (right), which are again somewhat smaller than the values used for the continuum model.

\section{B. $500 \mathrm{~K}$}

As shown in the middle column of Fig 1 shape oscillations of a type similar to those seen at $700 \mathrm{~K}$ are observed also in KMC simulations at $500 \mathrm{~K}$. Apart from the reduced amplitude of shape fluctuations, which is due to the larger island size, the main difference between the left and middle columns is a pronounced elongation of the island shape in all phases of the oscillation at $500 \mathrm{~K}$.

This elongation 29 shows up also in the stationary shapes which are characteristic of smaller islands and weaker bias (Fig 5 . A quantitative evaluation of the behavior of the aspect ratio of stationary islands as a function of island size and bias strength is shown in Fig 6 The aspect ratio is seen to increase with increasing island

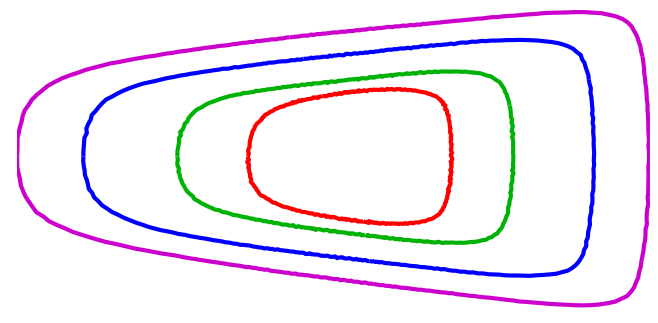

FIG. 5: Time-averaged stationary shapes for KMC islands of size $S=500,1000,2000$ and 3000 atoms at $T=500 \mathrm{~K}$ and $E_{\text {bias }}=10^{-3} \mathrm{eV}$. For this set of parameters oscillatory shape evolution sets in at $S=4000$, see the middle column of Fig [1
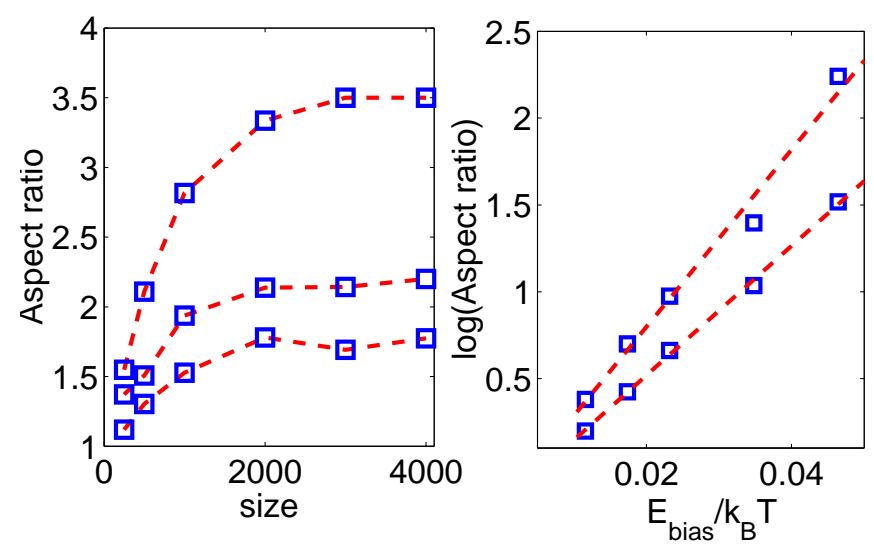

FIG. 6: Island aspect ratio, defined as the ratio of the maximal extensions along and perpendicular to the field, for stationary islands obtained from KMC simulations at 500 K. Left: the aspect ratio as a function of island size with $E_{\text {bias }}=7.5 \times 10^{-4} \mathrm{eV}, 1.0 \times 10^{-3} \mathrm{eV}$, and $1.5 \times 10^{-3} \mathrm{eV}$ (from bottom to top). The dashed lines are guides to the eye. Right: the logarithm of the aspect ratio as a function of bias for $S=1000$ (lower line) and $S=4000$ (upper line). The dashed lines are linear fits to the data with the slopes 37 and 50 for $S=1000$ and $S=4000$, respectively.

size up to some upper limit where it saturates. Increasing the island size further eventually leads to the onset of oscillations, as shown in the middle column of Fig [1 at other parameter values island breakup may also result. As was described above, during an oscillation the front edge of the island splits up into two parts, one of which advances while the other one stays behind. As a consequence, the island width is halved and the aspect ratio doubled at the point of maximal elongation. Between oscillations the aspect ratio relaxes back to a value that coincides with the saturation value for large stationary islands shown in the left panel of Fig [ In this sense the onset of oscillations can be seen as an attempt of the island to increase its aspect ratio beyond the maximal value that can be realized by stationary shapes.

The right panel of Fig 6 shows that the dependence of the island shape on the strength of the electromigration 
bias is fundamentally different from the dependence on island size: Rather than saturating, the aspect ratio of stationary islands continues to grow with increasing bias, and no onset of oscillations is observed at least for islands sizes $S<4000$. The inequivalence between size and bias dependence implies the breakdown of the continuum theory, which predicts that the islands shape should depend only on the dimensionless parameter $S / l_{E}^{2} \sim S E_{\text {bias. }}$. To give a quantitative example, the data in Fig 6 show that an island of size $S=2000$ driven by a bias force of strength $E_{\text {bias }}=10^{-3} \mathrm{eV}$ attains a stationary aspect ratio $A \approx 2.2$, whereas for $S=1000$ and $E_{\text {bias }}=2 \times 10^{-3}$ $\mathrm{eV}$ the aspect ratio is twice as large. Within the continuum theory, the two parameter combinations should lead to identical shapes. Indeed, continuum calculations carried out at $500 \mathrm{~K}$ fail to reproduce the elongated shapes seen in the KMC simulations. Instead, at the dimensionless radius $\hat{R} \approx 1.2$ corresponding to the cases described above, one finds stationary shapes with an aspect ratio near unity. Larger islands undergo OS motion for $2.1<\hat{R}<2.2$ and break up for $\hat{R} \geq 2.2$.

Quantitatively the data for the aspect ratio $A$ in the right panel of Fig [6]roughly follow an exponential relation

$$
A \sim e^{\alpha E_{\text {bias }} / k_{\mathrm{B}} T}
$$

where $\alpha=37$ and 50 for $S=1000$ and 4000, respectively. While the origin of this behavior so far eludes us, it is interesting to note that, in order of magnitude, $\alpha \sim \sqrt{S}$. This indicates that the relevant energy scale governing the behavior of the aspect ratio may be related to the energy gain associated with moving a row of atoms (of length $\sim \sqrt{S}$ ) by one lattice spacing in the direction of the electromigration force.

\section{CONCLUSIONS}

The most important outcome of our study is the observation of oscillatory island shape evolution, a phenomenon first predicted on the basis of continuum theory ${ }^{16.17}$, within a realistic KMC model of the $\mathrm{Cu}(100)$ surface. Since the KMC model has been previously validated against experimental observations ${ }^{2}$, we can now confidently pin down the conditions under which electromigration-driven shape oscillations should be realizable in the laboratory. Assuming that a bias strength of the order of $E_{\text {bias }}=10^{-5} \mathrm{eV}$ can be achieved experimentally 13 , extrapolation of the KMC results shown in Fig 3 indicate that oscillatory behavior of the kind shown in Fig 1 should emerge at $700 \mathrm{~K}$ for islands containing about $2 \times 10^{5}$ atoms, corresponding to an effective radius of approximately $90 \mathrm{~nm}$. The period of oscillations is expected to lie between $2 \mathrm{~s}$ and $40 \mathrm{~s}$, depending on which value of $z$ is used in the relation (11).
It is instructive to compare the modes of island migration observed in the present study of a surface with fourfold crystalline anisotropy to previous work on the continuum model, where a sixfold anisotropy of the step atom mobility was assumed ${ }^{16}$. Generally speaking, we find that the parameter regime in which complex motion occurs (the OO phase discussed above in Sect 【II) is less extended, and islands are more prone to breakup than in the sixfold symmetric case. Also, the spontaneous symmetry breaking associated with the OS phase (which could be detected here only in the deterministic continuum dynamics) is more pronounced in the model with sixfold anisotropy, in the sense that the angle between the direction of motion and the direction of the force is much larger ${ }^{16}$. But it is reassuring that the precise form of the anisotropies of stiffness and edge atom mobility seems to be inessential for the basic phenomenon of oscillatory shape evolution to emerge.

A detailed explanation of the breakdown of continuum scaling and the associated elongation of island shapes observed in the simulations at $500 \mathrm{~K}$ is lacking at present, and we can offer only some rather general remarks. In previous studies of island shape evolution deviations from the continuum approach were typically found when the characteristic size of the islands becomes comparable to the spacing $l_{k}$ between thermal kinks 8.10 .11 . Since electromigration-dominated dynamics sets in at island sizes comparable to $l_{E}$, it is instructive to consider the ratio

$$
\frac{l_{k}}{l_{E}}=\sqrt{\frac{E_{\mathrm{bias}}}{2 k_{\mathrm{B}} T}} e^{\epsilon / 2 k_{\mathrm{B}} T},
$$

which is seen to increase with decreasing temperature and increasing bias strength. For the standard value $E_{\text {bias }}=10^{-3} \mathrm{eV}$ used in our work, the ratio (13) increases from 0.27 at $700 \mathrm{~K}$ to 0.49 at $500 \mathrm{~K}$, which makes it plausible that the continuum description may become questionable in the latter case. According to this criterion, for an experimentally realizable bias strength of $E_{\text {bias }}=10^{-5} \mathrm{eV}$ the breakdown would be shifted to much lower temperatures, and the continuum description should remain valid down to room temperature and below.

\section{Acknowledgements}

This work was supported by DFG within project KR 1123/1-2, by the Academy of Finland within its Center of Excellence program (COMP), and by the EU within project MagDot. We thank T. Ala-Nissila for fruitful discussions. JK is grateful to $\mathrm{H}$. Mehl for useful correspondence, and to the Laboratory of Physics at TKK for its gracious hospitality during the early stages of this project. 
* krug@thp.uni-koeln.de

1 H.C. Jeong, E.D. Williams, Surf. Sci. Rep. 34, 171 (1999).

2 M. Giesen, Progr. Surf. Sci. 68, 1 (2001).

3 T. Michely, J. Krug, Islands, Mounds and Atoms. Patterns and Processes in Crystal Growth Far from Equilibrium. (Springer, Berlin 2004).

${ }^{4}$ O. Pierre-Louis, C.R. Physique 6, 11 (2005).

5 S. Kodambaka, S.V. Khare, I. Petrov, J.E. Greene, Surf. Sci. Rep. 60, 55 (2006).

6 A. Voigt (ed.), Multiscale modeling of epitaxial growth, ISNM Vol. 149 (Birkhäuser, Basel, 2005).

7 J. Krug, Ref.6, p. 69.

8 N. Combe, H. Larralde, Phys. Rev. B 62, 16074 (2000).

${ }^{9}$ O. Pierre-Louis, Phys. Rev. Lett. 87, 106104 (2001).

10 D.-J. Liu, J.W. Evans, Phys. Rev. B 66, 165407 (2002).

11 J.L. Iguain, L.J. Lewis, Phys. Rev. B 68, 195407 (2003).

12 J.-J. Métois, J.-C. Heyraud, A. Pimpinelli, Surf. Sci. 420, 250 (1999); A. Saúl, J.-J. Métois, A. Ranguis, Phys. Rev. B 65, 075409 (2002).

13 H. Mehl, O. Biham, O. Millo, M. Karimi, Phys. Rev. B 61, 4975 (2000).

14 O. Pierre-Louis, T.L. Einstein, Phys. Rev. B 62, 13697 (2000).

15 Z. Suo, in: Comprehensive Structural Integrity, Vol. 8: Interfacial and Nanoscale Failure, W. Gerberich, W. Yang, Eds. (Elsevier, Amsterdam 2003), p. 265.

16 P. Kuhn, J. Krug, F. Hausser, A. Voigt, Phys. Rev. Lett.
94, 166105 (2005).

17 P. Kuhn, J. Krug, in Ref.6, p. 159.

18 J. Heinonen, I. Koponen, J. Merikoski, T. Ala-Nissila, Phys. Rev. Lett. 82, 2733 (1999).

19 J. Merikoski, I. Vattulainen, J. Heinonen, and T. AlaNissila, Surf. Sci. 387, 167 (1997); J. Merikoski and T. Ala-Nissila, Phys. Rev. B 52, R8715 (1995).

20 A. B. Bortz, M. H. Kalos, and J. L. Lebowitz, J. Comput. Phys. 17, 10 (1975); M. Kotrla, Comput. Phys. Commun. 97, 82 (1995).

21 J. L. Blue, I. Beichl, and F. Sullivan, Phys. Rev. E 51, R867 (1995).

22 M. Rusanen, I.T. Koponen, J. Heinonen, T. Ala-Nissila, Phys. Rev. Lett. 86, 5317 (2001).

23 S. Dieluweit, H. Ibach, M. Giesen, T.L. Einstein, Phys. Rev. B 67, 121410(R) (2003).

24 T.J. Stasevich, T.L. Einstein, R.K.P. Zia, M. Giesen, H. Ibach, F. Szalma, Phys. Rev. B 70, 245404 (2004).

25 M. Giesen, S. Dieluweit, J. Mol. Cat. A 216, 263 (2004).

26 C. Tao, T.J. Stasevich, T.L. Einstein, E.D. Williams, Phys. Rev. B 73, 125436 (2006).

27 J. Krug, H.T. Dobbs, S. Majaniemi, Z. Phys. B 97, 281 (1995).

28 J. Kallunki, J. Krug, Surf. Sci. 523, L53 (2003).

29 Similar behavior was observed by H. Mehl in simulations of vacancy islands (private communication). 